\title{
On the Statistical Mechanics of the Gauge Invariant Ising Model
}

\author{
R. Marra ${ }^{1}$ and S. Miracle-Sole ${ }^{2}$ \\ ${ }^{1}$ Institute of Physics, University of Salerno, I-84100 Salerno, Italy \\ ${ }^{2}$ Centre de Physique Théorique, C.N.R.S., F-13288 Marseille, France
}

\begin{abstract}
Some results on the phase structure of the gauge invariant Ising model are derived by using convergent expansions.
\end{abstract}

\section{Introduction and Statement of the Results}

The gauge invariant Ising model, with which we shall be concerned in this paper, is one of the Wegner's generalized Ising models [1] and can be viewed as a lattice Higgs model, locally gauge invariant under the group $\mathbb{Z}_{2}$.

One believes that this model, appearing then as one of the simplest models for a gauge theory on a lattice according to Wilson's ideas $[2,3]$, can already be useful to obtain some insight into the physics of gauge theories at least in the abelian case.

A general outline and results on such lattice theories, in relation with the present study, may be found in $[4,5]$.

From certain extrapolation arguments briefly reported in the next section, the following peculiar phase structure is conjectured for this system $[1,6]$ : a critical line, at which a second order phase transition would take place, separates two regions in the plane of the coupling parameters $\left(\beta_{p}, \beta_{l}\right)$, corresponding to the pure phase domains in the phase diagram of the system. One expects also that a qualitative different particle behaviour marks the difference between the two regions, which could correspond to a region of particle confinement and a region where separated charge excitations are allowed.

Our purpose here is to analyze the first mentioned conjecture concerning the phase structure of the system by the use of convergent expansions, a very familiar technique in statistical mechanics. For the sake of definiteness we shall consider the case of a 3-dimensional lattice.

According to the conjecture and the particular form of the expected phase diagram we determine two regions I and II in the plane $\left(\beta_{p}, \beta_{l}\right)$ where the corresponding expansions converge (Fig. 1). 


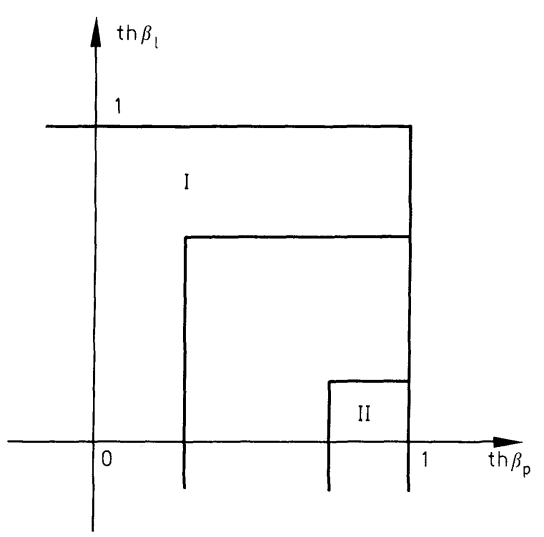

Fig. 1. The analyticity regions

These regions are such that:

I) If $\left|\beta_{p}\right|$ is sufficiently small or if $\beta_{p} \geqq 0$ and $\left|\beta_{l}\right|$ is sufficiently large the point $\left(\beta_{p}, \beta_{l}\right)$ belongs to the region $I$.

II) If $\beta_{p}$ is positive and sufficiently large and $\left|\beta_{l}\right|$ is sufficiently small the point $\left(\beta_{p}, \beta_{l}\right)$ belongs to the region II.

As a consequence of these facts the analyticity of the free energy and the unicity and analyticity of the translation invariant Gibbs state follow in regions I and II.

The convergent expansions may also be a useful tool for studying the probabilistic structure of the pure phases in the analyticity regions I and II. Such a study would be of interest in connection with the second mentioned conjecture on the particle behaviour of the system.

\section{Definitions and Preliminary Remarks}

The model can be described as follows : the configuration variables $\theta(x)$ and $j\left(x, x^{\prime}\right)$ take values in $\mathbb{Z}_{2}=\{-1,+1\}$ and are respectively indexed by the sites $x \in \mathscr{L}$ and the links $\left(x, x^{\prime}\right) \subset \mathscr{L}$ (pairs of nearest neighbour sites) of a cubic lattice $\mathscr{L}$. The hamiltonian is

$$
H_{\Lambda}(\theta, j)=-\left\{\beta_{l} \sum_{\left(x, x^{\prime}\right) \subset \mathscr{L}} j\left(x, x^{\prime}\right) \theta(x) \theta\left(x^{\prime}\right)+\beta_{p} \sum_{P \subset A} j(P)\right\}
$$

where $\Lambda$ is a finite box, $\beta_{l}$ and $\beta_{p}$ are the coupling parameters, $P \subset A$ denotes the set of plaquettes (elementary squares of the lattice) contained in $\Lambda$, and $j(P)$ is the product of the $j$ 's corresponding to the links of the plaquette $P$.

To describe the infinite system one introduces the thermodynamic free energy and the Gibbs states which are probability measures on the space of configurations verifying the Dobrushin-Lanford-Ruelle equations [7].

The system is invariant under the local $Z_{2}$ transformations (gauge transformations)

$$
\theta(x) \rightarrow k(x) \theta(x) \quad j\left(x, x^{\prime}\right) \rightarrow k(x) j\left(x, x^{\prime}\right) k\left(x^{\prime}\right)
$$


where $k(x)$ is any $\mathbb{Z}_{2}$ valued function on the lattice sites. Their local character implies that not only the free energy but also the Gibbs states are gauge invariant.

As any gauge invariant function of the configuration depends only on the reduced variables $\tau\left(x, x^{\prime}\right)=\theta(x) j\left(x, x^{\prime}\right) \theta\left(x^{\prime}\right)$ one can reinterpret the model as having only configuration variables $\tau\left(x, x^{\prime}\right)$ and hamiltonian

$$
H_{\Lambda}(\tau)=-\left\{\beta_{l} \sum_{\left(x, x^{\prime}\right) \subset A} \tau\left(x, x^{\prime}\right)+\beta_{p} \sum_{P \subset A} \tau(P)\right\}
$$

It will be useful to notice that model (3) at $\beta_{l}=0$ has again a local $\mathbb{Z}_{2}$ invariance property.

Model (3) is actually one of the models introduced by Wegner. We now point out some relevant properties of it, most of them already stated in [1].

In the 3-dimensional case the model is self-dual, namely (up to an unimportant additive term) the free energy is invariant under the transformation $\left(\beta_{p}, \beta_{l}\right) \rightarrow\left(\beta_{p}^{*}, \beta_{l}^{*}\right)$ where

$$
\text { th } \beta_{p}^{*}=\exp -2 \beta_{l} \quad \text { th } \beta_{l}^{*}=\exp -2 \beta_{p}
$$

This symmetry property extends in an appropriate way to the correlation functions with particular boundary conditions.

If $\beta_{l}=0$ the model (also called the pure gauge model), with some coupling $\beta_{p}$ is related by duality to the usual 3-dimensional Ising model with coupling $\beta^{*}=-(1 / 2) \log \operatorname{th} \beta_{p}$. It has therefore a critical point at a definite value $\beta_{p}^{c}$ of the coupling. A non local order parameter connected with the average $\left\langle\tau_{\Gamma}\right\rangle$, where $\tau_{\Gamma}$ is the product of $\tau$ 's associated to links forming a closed curve $\Gamma$ was introduced in [1] to characterize this phase transition. It was stated that this average $\left\langle\tau_{\Gamma}\right\rangle$ has an exponential decay ruled by the area enclosed by $\Gamma$ below $\beta_{p}^{c}$ and by the perimeter of $\Gamma$ above $\beta_{p}^{c}$. This order parameter, which corresponds in this model to the Wilson loop integral, is related to the confinement problem [2] and a proof of the mentioned behaviour has been given in [8] for $\beta_{p}$ out of a small interval around the critical value.

By duality the expectation $\left\langle\tau_{\Gamma}\right\rangle$ is related to the surface tension [9] of the 3dimensional Ising model at the coupling value $\beta^{*}$. The above remarks prove then that the surface tension of the Ising model is strictly positive at low temperature (large $\beta^{*}$ ) where in the dual gauge model one has a surface behaviour of the order parameter and that the surface tension is zero at high temperature (small $\beta^{*}$ ) where perimeter behaviour holds.

If $\beta_{l}$ is strictly positive the behaviour of $\left\langle\tau_{\Gamma}\right\rangle$ is always ruled by the perimeter $|\Gamma|$. This fact can easily be proved by means of the Griffiths-Kelly-Sherman inequalities [10] which show that $\left\langle\tau_{\Gamma}\right\rangle$, at some positive $\beta_{p}$ and $\beta_{l}$, is larger than $\left\langle\tau_{\Gamma}\right\rangle$ at $\beta_{p}=0$ and the same $\beta_{l}$. But at this point one finds $\left\langle\tau_{\Gamma}\right\rangle=\left(\operatorname{th} \beta_{l}\right)^{|\Gamma|}$, showing the perimeter behaviour of $\left\langle\tau_{\Gamma}\right\rangle$ whenever $\beta_{l}>0$.

Since $\left(\beta_{l}=0, \beta_{p}^{c}\right)$ is a critical point it follows by the self-dual property of the model that also the point $\left(\beta_{l}=\beta_{l}^{*}\left(\beta_{p}^{c}\right), \beta_{p}=\infty\right)$ is critical. Hence the conjecture that these critical points are joined by a whole critical line which divides the plane $\left(\beta_{p}, \beta_{l}\right)$ in two regions; conjecture which is also supported in part by mean field calculations [1] near the point $\left(\beta_{l}=0, \beta_{p}^{c}\right)$. 


\section{Convergent Expansions}

Actually the existence of the analyticity region I has already been proved and the corresponding convergent expansions have been treated in [6] and [11] for more general lattice gauge theories. We shall give, however, in our case, an alternative proof based on known results in statistical mechanics.

We first remark that in (3) $\beta_{l}$ plays the role of a magnetic field. Therefore the known results on the high temperature behaviour of lattice systems [10] imply that if $\left|\beta_{p}\right|$ is sufficiently small the free energy is an analytic function and the Gibbs state is unique and analytic for all values of $\beta_{l}$. From the self-dual property of the model this initial analyticity region is extended to a region invariant under (4) which has the properties stated in the introduction for the region I (we also use the symmetry $\beta_{l} \rightarrow-\beta_{l}$ ). In this region the free energy is still analytic.

The expansion which corresponds to the weak coupling region is obtained from the following known formula for the partition function. Denote by $N_{l}(\Lambda)$ the number of links and by $N_{p}(\Lambda)$ the number of plaquettes contained in $\Lambda$ and let $R_{\Lambda}=\left(\operatorname{ch} \beta_{l}\right)^{N_{l}(\Lambda)}\left(\operatorname{ch} \beta_{p}\right)^{N_{p}(\Lambda)}$ Then

$$
\begin{aligned}
Z_{\Lambda} & =\sum_{\{\tau= \pm 1\}} \exp \left\{\beta_{l} \sum_{\left(x, x^{\prime}\right) \subset A} \tau\left(x, x^{\prime}\right)+\beta_{p} \sum_{P \subset A} \tau(P)\right\} \\
& =R_{\Lambda} \sum_{\{\tau= \pm 1\}} \prod_{\left(x, x^{\prime}\right) \subset \Lambda}\left(1+\operatorname{th} \beta_{l} \tau\left(x, x^{\prime}\right)\right) \prod_{P \subset A}\left(1+\operatorname{th} \beta_{p} \tau(P)\right) \\
& =R_{\Lambda} \sum_{S \subset A}\left(\operatorname{th} \beta_{p}\right)^{|S|}\left(\operatorname{th} \beta_{l}\right)^{|\partial S|}
\end{aligned}
$$

where $S \subset \Lambda$ is the family of all subsets $S$ of the set of plaquettes contained in $\Lambda$. A set of plaquettes can be considered as a surface of area $|S|$, and $\partial S$ denotes the boundary of this surface, which is the set of closed circuits formed by links at which an odd number of plaquettes of $S$ occurs ( $|\partial S|$ is the number of links).

We do not enter in details of the convergent expansion for the region I, which follows from (5) by standard arguments, but prefer to come to the discussion of a new expansion which will be the appropriate expansion for proving analyticity in the region II.

Given a configuration we consider the set of plaquettes at which $\tau(P)=-1$. We introduce the dual lattice $\mathscr{L}^{*}$ which is a cubic lattice the links of which cut perpendicularly the plaquettes of $\mathscr{L}$ in such a way that the centres of the plaquettes coincide with the middle points of the links. We denote by $\Lambda^{*}$ the subset of $\mathscr{L}^{*}$ formed by the links which cut the plaquettes of $\mathscr{L}$ contained in $\Lambda$. Call $C$ the set of links in $\mathscr{L}^{*}$ intersecting the plaquettes of $\mathscr{L}$ at which $\tau(P)=-1$. Since $\prod_{P \in \mathscr{K}} \tau(P)=1$ for any elementary cube $\mathscr{K}$ of $\mathscr{L}$ at every site of the lattice $\mathscr{L}^{*}$ only an even number (or zero) of links of $C$ occur (for an appropriate boundary condition). This means that $C$ is a set of closed circuits of links on $\mathscr{L}^{*}$. Let $Q_{\Lambda}=\exp \beta_{p} N_{p}(\Lambda)$ $\left(\operatorname{ch} \beta_{l}\right)^{N_{l}(\Lambda)}$ and denote by $|C|$ the number of links of $C$. The partition function can then be written

$$
Z_{\Lambda}=Q_{\Lambda} \sum_{C \subset \Lambda^{*}} \exp \left(-2 \beta_{p}|C|\right) \sum_{\{\tau= \pm 1\}}^{\prime} \prod_{\left(x, x^{\prime}\right) \subset \Lambda}\left(1+\operatorname{th} \beta_{l} \tau\left(x, x^{\prime}\right)\right)
$$


where the sum $\sum^{\prime}$ is restricted to the configurations $\{\tau= \pm 1\}$ such that $\tau(P)=-1$ if $P$ intersects $C$ and $\tau(P)=1$ if $P$ does not intersect $C$.

Develop now the product $\prod\left(1+\operatorname{th} \beta_{l} \tau\left(x, x^{\prime}\right)\right)$ and observe that only the terms of the form $\prod_{\left(x, x^{\prime}\right) \subset C^{\prime}} \operatorname{th} \beta_{l} \tau\left(x, x^{\prime}\right)$ where $C^{\prime}$ is a set of closed circuits of links in $\mathscr{L}$ give a non-zero contribution. Then $\prod_{\left(x, x^{\prime}\right) \subset C^{\prime}} \tau\left(x, x^{\prime}\right)=\prod_{P \in S} \tau(P)$ for any set of plaquettes such that $\partial S=C^{\prime}$ and hence its value +1 or -1 is determined. We denote by $n\left(C, C^{\prime}\right)$ this number and then

$$
Z_{\Lambda}=Q_{\Lambda} \sum_{C \subset \Lambda^{*}} \sum_{C^{\prime} \subset \Lambda} \exp \left(-2 \beta_{p}|C|\right)\left(\operatorname{th} \beta_{l}\right)^{\left|C^{\prime}\right|} n\left(C, C^{\prime}\right)
$$

The expressions (5) and (6) show two geometrical aspects of the interaction defining the system. We may also notice that from any of those the duality properties mentioned in Sect. 2 easily follow.

We now modify slightly the expression (6). For this, we decompose the set $C$ into a set of non-intersecting closed curves of adjacent links in $\mathscr{L}^{*}$, by smoothing if necessary the corners of the circuits in a specified way at each lattice site. In the same way we decompose the set $C^{\prime}$ in $\mathscr{L}$. We obtain then

$$
\begin{aligned}
Z_{\Lambda}= & Q_{\Lambda} \sum_{\left\{\gamma_{1}, \ldots, \gamma_{n}\right\} \subset \Lambda^{*}\left\{\gamma_{1}^{\prime}, \ldots, \gamma_{m}^{\prime}\right\} \subset \Lambda} \exp \left\{-2 \beta_{p}\left(\left|\gamma_{1}\right|+\ldots+\left|\gamma_{n}\right|\right)\right. \\
& \times\left(\operatorname{th} \beta_{l}\right)^{\left|\gamma^{\prime} 1\right|+\ldots+\left|\gamma_{m}^{\prime}\right|} \prod_{i=1}^{n} \prod_{j=1}^{m} n\left(\gamma_{i}, \gamma_{j}^{\prime}\right)
\end{aligned}
$$

where $\left\{\gamma_{1}, \ldots, \gamma_{n}\right\} \subset \Lambda^{*}$ and $\left\{\gamma_{1}^{\prime}, \ldots, \gamma_{m}^{\prime}\right\} \subset \Lambda$ mean the family of all sets of possible non-intersecting curves in $\mathscr{L}^{*}$ and $\mathscr{L}$.

The next step is to factorize the terms appearing in the partition function. In order to do this the following definitions are introduced.

To any set of non-intersecting closed curves in $\mathscr{L}$ and $\mathscr{L}^{*}$ an abstract graph is associated in the following way. The closed curves of the set are in one-to-one correspondence with the vertices of the graph. Two vertices of the graph are joined by a line if and only if they are associated to a curve $\gamma^{\prime}$ in $\mathscr{L}$ and a curve $\gamma$ in $\mathscr{L}^{*}$ such that $n\left(\gamma, \gamma^{\prime}\right)=-1$.

We define a contour $\Gamma=\left\{\gamma_{1}, \ldots, \gamma_{n}, \gamma_{1}^{\prime}, \ldots, \gamma_{m}^{\prime}\right\}$ as a set of non-intersecting closed curves $\left\{\gamma_{1}, \ldots, \gamma_{n}\right\} \subset \mathscr{L}^{*}$ and $\left\{\gamma_{1}^{\prime}, \ldots, \gamma_{m}^{\prime}\right\} \subset \mathscr{L}$ such that the graph associated to $\Gamma$ is a connected graph. A contour can also be reduced to just one curve in $\mathscr{L}$ or in $\mathscr{L}^{*}$. The following weight

$$
\mu(\Gamma)=\exp \left\{-2 \beta_{p}\left(\left|\gamma_{1}\right|+\ldots+\left|\gamma_{n}\right|\right\}\left(\operatorname{th} \beta_{l}\right)^{\left|\gamma_{1}^{\prime}\right|+\ldots+\left|\gamma_{m}^{\prime}\right|} \prod_{i=1}^{n} \prod_{j=1}^{m} n\left(\gamma_{i}, \gamma_{j}^{\prime}\right)\right.
$$

is assigned to each contour $\Gamma$.

Two contours $\Gamma_{1}$ and $\Gamma_{2}$ are said to be compatible, and we write $\Gamma_{1} \sim \Gamma_{2}$, if the curves of $\Gamma_{1}$ and of $\Gamma_{2}$ do not intersect and if the sets $\Gamma_{1}$ and $\Gamma_{2}$ define disjoint subgraphs in the graph associated to $\Gamma_{1} \cup \Gamma_{2}$.

The partition function becomes then

$$
Z_{\Lambda}=\sum_{\substack{\left\{\Gamma_{1}, \ldots, \Gamma_{n}\right\} \subset \Lambda \cup \Lambda^{*} \\ \Gamma_{2} \sim \Gamma_{J}}} \mu\left(\Gamma_{1}\right) \ldots \mu\left(\Gamma_{n}\right)
$$


where $\Gamma_{i} \sim \Gamma_{j}$ indicates that the sum runs over all arrangements of mutually compatible contours.

At this point, the system can be studied by a method analogous to the method developped by Minlos and Sinai for the study of the Ising model at low temperature [12] (although $\mu(\Gamma)$ has not the meaning of a probability, being only a weight contribution to the partition function).

We introduce the functions

$$
\varrho_{\Lambda}\left(\left\{\Gamma_{1}, \ldots, \Gamma_{n}\right\}\right)=Z_{\Lambda}^{-1} \sum_{\substack{\left\{\bar{\Gamma}_{1}, \ldots, \bar{\Gamma}_{m\}}\right\} \\ \Gamma_{2} \sim \bar{\Gamma}_{J}, \bar{\Gamma}_{i} \sim \bar{\Gamma}_{J}}} \mu\left(\Gamma_{1}\right) \ldots \mu\left(\Gamma_{n}\right) \mu\left(\bar{\Gamma}_{1}\right) \ldots \mu\left(\bar{\Gamma}_{m}\right)
$$

defined on the families of compatible contours $\left\{\Gamma_{1}, \ldots, \Gamma_{n}\right\} \subset \Lambda \cup \Lambda^{*}$. These functions verify the following system of recursive equations

$$
\varrho_{1}\left(\left\{\Gamma_{1}, \Gamma_{2}, \ldots, \Gamma_{n}\right\}\right)=\left(\mu ( \Gamma _ { 1 } ) \left\{\varrho_{\Lambda}\left(\left\{\Gamma_{2}, \ldots, \Gamma_{n}\right\}\right)+\sum_{\substack{\left\{\bar{\Gamma}_{1}, \ldots, \bar{\Gamma}_{m}\right\} \subset A \cup \Lambda^{*} \\ \bar{\Gamma}_{2} \sim \bar{\Gamma}_{j} \\ \bar{\Gamma}_{i} \sim \Gamma_{j} j=2, \ldots, n \\ \bar{\Gamma}_{i} \nsim \Gamma_{1}}}(-1)^{m} \varrho_{\Lambda}\left(\left\{\Gamma_{2}, \ldots, \Gamma_{n}, \bar{\Gamma}_{1}, \ldots, \bar{\Gamma}_{m}\right\}\right)\right.\right.
$$

where $\bar{\Gamma}_{i} \nsim \Gamma_{1}$ means that $\bar{\Gamma}_{i}$ is not compatible with $\Gamma_{1}$ for any $i=1, \ldots, m$. This system of equations is analogous to the Minlos-Sinai contour equations for the Ising model and can be deduced similarly. It can also be interpreted, as in the case considered in [12], as a nonhomogeneous integral equation in an appropriate Banach space, the elements of which are the sets of functions $\varrho_{\Lambda}\left(\left\{\Gamma_{1}, \ldots, \Gamma_{n}\right\}\right)$, $n=1,2, \ldots$.

By remembering the definition given above of compatible contours and making use of the facts that through a fixed point in a 3-dimensional lattice it is impossible to construct more than $5^{l}$ different curves of length $l(l \geqq 4$ if the curve is closed) and that the minimal surface having as the boundary a fixed curve of length $l$ has an area less than $l^{2}$, it comes that the following series

$$
\mu\left(\Gamma_{1}\right) \sum_{\Gamma \nsim \Gamma_{1}} \mu(\Gamma)
$$

is convergent whenever the two conditions

$$
\begin{array}{r}
\sum_{l=4}^{\infty} 5^{l} l^{2} \exp \left\{-2 \beta_{p} l\right\}<1 \\
\sum_{l=4}^{\infty} 5^{l} l^{2}\left(\operatorname{th} \beta_{l}\right)^{l}<1
\end{array}
$$

are satisfied. Moreover (9) has a bound $\varepsilon\left(\beta_{p}, \beta_{l}\right)$ which tends to zero when $\beta_{p} \rightarrow \infty$ and $\beta_{l} \rightarrow 0$.

This is enough to guarantee that the kernel of the integral equation has a norm strictly less than 1 provided that $\beta_{p}$ is sufficiently large and $\beta_{l}$ is sufficiently small, that is if $\left(\beta_{p}, \beta_{l}\right)$ belongs to region II. From this it follows that the limits $\Lambda \rightarrow \infty$ of the functions $\varrho_{A}$ exist and are analytic with respect to $\beta_{p}$ and $\beta_{l}$ in the region II, and also that the free energy is analytic in this region (the detailed arguments can be found in [10]). 
We remark that from expression (5) a treatment with a similar system of equations could be developped in the weak coupling region. The role of contours is played in this case by open surfaces made of the plaquettes.

\section{Conclusion}

In this section we conclude the proof of the following proposition.

Proposition. In the regions $I$ and II defined in the introduction the free energy $f\left(\beta_{p}, \beta_{l}\right)$ of the system is an analytic function of its arguments. Moreover in this region the translation invariant Gibbs state is unique and analytic with respect to $\beta_{p}$ and $\beta_{l}$.

The analyticity of the free energy in both regions has been proved in the last section, and also the analyticity of the Gibbs state corresponding to a particular boundary condition. Although one could probably use the duality arguments in region $I$ and the contour techniques in region II to prove the unicity of the translation invairant Gibbs state it would be a delicate problem which requires a treatment of the different boundary conditions. The following remark based on Lebowitz's results [13] gives a direct proof of this result: if at the point $\beta_{p} \geqq 0$, $\beta_{l} \geqq 0$ the first derivatives of $f\left(\beta_{p}, \beta_{l}\right)$ exist then the translation invariant Gibbs state is unique. This remark is proved by noticing that if $\beta_{l}>0,\left\langle\tau\left(x, x^{\prime}\right)\right\rangle$ is larger than zero, by Griffiths-Kelly-Shermann inequalities [10] and unique for the translation invariant Gibbs states because $\partial f / \partial \beta_{l}$ exists. Then the argument in [13] shows that all translation invariant correlation functions are unique. If $\beta_{l}=0$ and $\beta_{p}>0$ then $\langle\tau(P)\rangle>0$ and unique since $\partial f / \partial \beta_{p}$ exists. Then the same arguments imply that all translation invariant correlation functions of the variables generated by products of $\tau(P)$ are unique. Since the non gauge-invariant functions are zero at $\beta_{l}=0$ the unicity of the state follows.

In order to simplify the exposition we made explicit use of particular properties of the model in 3-dimensions, but the results above can be generalized to any dimension by similar arguments.

Acknowledgments. It is a pleasure to thank M. Cassandro, G. Gallavotti and F. Guerra for useful discussions. One of us (S.M.S.) wishes to thank the members of the Istituto Matematico of the University of Rome for a very friendly hospitality.

\section{References}

1. Wegner, F.: J. Math. Phys. 12, 2259 (1971)

2. Wilson, K.G.: Phys. Rev. D 10, 2445 (1974)

3. Jaffe, A.: Lattice instantons: what they are and why they are important; de Angelis, G.F., de Falco, D., Guerra, E.: Gauge Fields on the lattice; Osterwalder, K., Seiler, E.: Lattice gauge theories; Frohlich, J.: Some frontiers in constructive quantum field theory and equilibrium statistical mechanics.

In : Mathematical problems in theoretical physics. Dell'Antonio, G.F., Doplicher, S., Jona-Lasinio, G., (eds.). Lecture notes in physics, Vol. 80. Berlin, Heidelberg, New York: Springer 1978

4. Balian, R., Drouffe, J.M., Itzykson, C. : Phys. Rev. D 10, 3376 (1974); D 11, 2098 (1975); and D 11, 2104 (1975)

5. Osterwalder, K., Seiler, E.: Ann. Phys. 110, 440 (1978) 
6. de Angelis, G.F., de Falco, D., Guerra, F., Marra, R.: Confinement as a problem in statistical mechanics. Preprint, University of Salerno (1978)

7. Lanford, O.E.: Entropy and equilibrium states in classical statistical mechanics. In: Statistical mechanics and mathematical problems. Lenard, A. (ed.). Lecture notes in physics Vol. 20. Berlin, Heidelberg, New York: Springer 1973

8. Gallavotti, G., Guerra, F., Miracle-Sole, S.: A comment to the talk by E. Seiler. In Mathematical problems in theoretical physics. Dell'Antonio, G.F., Doplicher, S., Jona-Lasinio, G. (eds.). Leçture notes in physics Vol. 80, Berlin, Heidelberg, New York: Springer 1978

9. Gruber, G., Hintermann, A., Messager, A., Miracle-Sole, S.: Commun. Math. Phys. 56, 147 (1977)

10. Ruelle, D.: Statistical mechanics, New York: Benjamin 1969

11. Israel, R.B., Nappi, C.R.: Quark confinement in the two-dimensional Lattice Higgs-Villain Model. Preprint, Harvard University (1978)

12. Minlos, R.A., Sinai, Ja G.: Trans. Moscow Math. Soc. 17, 237 (1967); 19, 121 (1968)

13. Lebowitz, J.L.: J. Stat. Phys. 16, 463 (1977)

Communicated by E. Lieb

Received August 17, 1978; in revised form February 28, 1979 